\title{
Erratum: Binding energies of trions and biexcitons in two-dimensional semiconductors from diffusion quantum Monte Carlo calculations [Phys. Rev. B 95, 081301(R) (2017)]
}

\author{
M. Szyniszewski, E. Mostaani, N. D. Drummond, and V. I. Fal'ko \\ (Received 24 August 2017; published 6 September 2017)
}

DOI: 10.1103/PhysRevB.96.119902

We have noticed a typographical error in the definition of the $r_{*}$ parameter below Eq. (2). The definition should be $r_{*}=2 \pi \kappa_{\perp} / \epsilon$. This change does not affect any of our results. To clarify, the values reported in Table I were calculated for free-standing monolayers for which $\epsilon=1$. 